\title{
Three-Dimensional Illusion Thermotics with Separated Thermal Illusions
}

\author{
Xiayao Peng and Run $\mathrm{Hu}^{*}$
}

Thermal camouflage is used to confront with infrared (IR) imaging by concealed or misled heat signature of the targets. Most previous conductive thermal camouflage only achieves the equivalent temperature profiles outside the illusion device, and the targets may still be observable from the z-plane imaging. The two-dimensional (2D) illusion thermotics was proposed to add the split thermal illusions inside the illusion device, which advances the thermal camouflage performance. But a drawback remains that the thermal illusions are not separated from the heat source, which only creates distorted heat signatures rather than camouflages the heat source. In this study, we develop the threedimensional (3D) illusion thermotics with separated thermal illusions to remove the drawback for perfect thermal camouflage performance. Finite-element simulations validate that although the real heat source is located at the bottom center, both symmetric and asymmetric thermal illusions can be generated on the top surface, misleading the number, size, and position information of the real heat source from the z-plane IR imaging and achieving perfect thermal camouflage performance. The 3D illusion thermotics may open avenue for more thermal functionalities with enhanced flexibility at the cost of enhanced complexity.

Keywords: Illusion thermotics; Thermal camouflage; Thermal metamaterials; Thermal illusions; Transformation thermotics

Received 29 July 2019, Accepted 20 September 2019

DOI: $10.30919 /$ esee 8 c326

\section{Introduction}

Inspired by the natural animals like chameleon and octopus that can change their skin color to blend themselves into the background environment adaptively, the multi-discipline camouflage technologies surge with a lot of applications in recent decade like soft robots, radar stealth, acoustic absorber, thermal reshaper, etc. ${ }^{1-7}$ Among them, the thermal camouflage means to render the target objects in another form from an infrared (IR) camera which converts the thermal radiation energy into temperature field with setting the calibrated emissivity. ${ }^{5,89}$ Since thermal radiation occurs everywhere and all the time to facilitate the IR detection even at night, the thermal camouflage technology, competing with IR detection, is beset with difficulties and challenges. ${ }^{5,9}$ For thermal camouflage, one possible solution is to create the fake temperature field to mislead the IR camera, and the essence is to manipulate heat flow directionally, which seems to disobey its diffusive nature. In the nature world, most materials are isotropic and we have been accustomed to feel the isotropic world and accept and regard the omnidirectional heat flow as a natural common sense. Actually, there are special circumstances. For instance, the axial and radial thermal conductivities of wood, silk, and polymer differ greatly, and the in-plane and out-of-plane thermal conductivities of graphite and boron nitride vary significantly. ${ }^{10-13}$ These anisotropic materials enable the directional heat flow, at least much easier than the isotropic materials. To design the required anisotropic materials for thermal camouflage, the

State Key Laboratory of Coal Combustion, School of Energy and Power Engineering, Huazhong University of Science and Technology, Wuhan 430074, China

*E-mail: hurun@hust.edu.cn transformation thermotics theory was proposed in last decade, which is extended from the counterpart transformation optics theory. ${ }^{14,15}$ In transformation thermotics, the isotropic virtual space is transformed into the anisotropic real space with maintaining the heat conduction governing equation from $\nabla(\kappa \cdot \nabla T)=0$ to $\nabla^{\prime}\left(\kappa^{\prime} \cdot \nabla^{\prime} T^{\prime}\right)=0$, giving rise to the transformed thermal conductivity $\kappa^{\prime}=\Lambda \kappa \Lambda^{\mathrm{T}} / \operatorname{det}(\boldsymbol{\Lambda})$, where $\boldsymbol{\Lambda}$ is the transformation Jacobian matrix as $\boldsymbol{\Lambda}=\partial\left(x^{\prime}, y^{\prime}, z^{\prime}\right) / \partial(x, y, z) .{ }^{14}$ Based on transformation thermotics, people have extended to the illusion thermotics to design the related illusion devices to move the target, or to change the target shape, or to design the equivalent elements, or to cloak the target, while maintaining the same temperature field outside the device..$^{5,8,16-27}$ Though the outside observes may be misled by the same outside temperature fields, it should be admitted that the targets are still observable in the two-dimensional (2D) IR images with distinct temperature difference. ${ }^{5,8,23}$ In our previous paper, we further advanced this problem by adding the splitting function of the target heat source while maintaining the same temperature field outside the device. ${ }^{5,16}$ Multiple illusion heat sources were created inside the device to mislead the IR detection of the right information in terms of the number, location, shape, and size of the real targets. However, one problem remains that due to the diffusive heat conduction from the real target, the target heat source and the illusion heat source seems to be "linked" with equivalent temperatures, thus the thermal illusions are not "separated", which deteriorates the splitting function more or less.

To further solve this problem, we develop the three-dimensional (3D) illusion thermotics to design illusion devices for thermal camouflage with separated thermal illusions. Detailed design process was introduced, and required thermal conductivity tensors are derived and discussed. Finite-element simulations were conducted to validate the $3 \mathrm{D}$ design with both symmetrical and asymmetric thermal illusions. 


\section{Methodology}

To start, we introduce the 3D transformation thermotics method briefly. Since the original heat source is located at the original point of the 3D Cartesian coordinate system, the conductive plate located at the semihemispherical space is divided into four rectangular subspaces. For simplification without loss of generality, here we take the quarter plate in the first quadrant as an example for the design demonstration as follows. Figure 1a shows the quarter plate in the real space where the real plate is denoted by the box $A^{\prime} B^{\prime} C^{\prime} D^{\prime} E^{\prime} F^{\prime} G^{\prime} H^{\prime}$ and the real heat source is denoted by the cuboid P'Q'R'S'T'U'V'W' respectively. Accordingly, in the virtual plate is denoted by the box ABCDEFGH and the illusion heat source is denoted by PQRSTUVW respectively as shown in Fig. 1b. The coordinate transformation happens within the whole box, thus the real plate and virtual plate share the same dimension of $L \times W \times H$. The dimension of the real heat source is $l^{\prime} \times w^{\prime} \times h^{\prime}$, and the dimension of the virtual heat source is $l \times w \times h$ respectively. The whole box is then divided into some tetrahedron regions except for the real and virtual heat sources. All the dimensions (plate, heat source, and tetrahedrons) can be adjusted by changing the positions and number of vertex coordinates. For instance, the green tetrahedrons in the real space are composed by points $E^{\prime} V^{\prime} G^{\prime} H^{\prime}$ and $E^{\prime} V^{\prime} G^{\prime} F$ ' respectively, and the corresponding tetrahedrons in the virtual space are composed by points EVGH and EVGF respectively. Once all the coordinates are preset in advance, the Jacobian matrix of the coordinate transformation can be calculated. Then according to the transformation thermotics, the thermal conductivities of the tetrahedron regions in the real space can be obtained, enabling the finite-elementmethod (FEM) simulation validations.

To calculate the anisotropic thermal conductivity, we use a linear coordinate mapping between the virtual and real spaces as

$$
\left[\begin{array}{c}
x_{n}^{\prime} \\
y_{n}^{\prime} \\
z_{n}^{\prime}
\end{array}\right]=\left[\begin{array}{llll}
\alpha_{n} & \beta_{n} & \gamma_{n} & \varphi_{n} \\
\mu_{n} & v_{n} & \varepsilon_{n} & \theta_{n} \\
\lambda_{n} & \sigma_{n} & \rho_{n} & \omega_{n}
\end{array}\right]\left[\begin{array}{c}
x_{n} \\
y_{n} \\
z_{n} \\
1
\end{array}\right]
$$

with the subscript $n$ denotes the sequence number of the tetrahedron region. Then the Jacobian matrix and the anisotropic thermal conductivity in each tetrahedron region are

$$
\mathbf{J}_{n}=\frac{\partial\left(x_{n}^{\prime}, y_{n}^{\prime}, z_{n}^{\prime}\right)}{\partial\left(x_{n}, y_{n}, z_{n}\right)}=\left[\begin{array}{ccc}
\alpha_{n} & \beta_{n} & \gamma_{n} \\
\mu_{n} & v_{n} & \varepsilon_{n} \\
\lambda_{n} & \sigma_{n} & \rho_{n}
\end{array}\right]
$$

and

$$
\kappa_{n}^{\prime}=\left[\begin{array}{ccc}
\kappa_{x x}^{\prime} & \kappa_{x y}^{\prime} & \kappa_{x z}^{\prime} \\
\kappa_{y x}^{\prime} & \kappa_{y y}^{\prime} & \kappa_{y z}^{\prime} \\
\kappa_{z x}^{\prime} & \kappa_{z y}^{\prime} & \kappa_{z z}^{\prime}
\end{array}\right]_{n}=\frac{\mathbf{J}_{n} \kappa_{0} \mathbf{J}_{n}{ }^{\mathrm{T}}}{\operatorname{det}\left(\mathbf{J}_{n}\right)}
$$

with $\kappa_{0}$ the thermal conductivity of the isotropic virtual plate and it is assumed as $1 \mathrm{~W} / \mathrm{m}-\mathrm{K}$ in following designs.
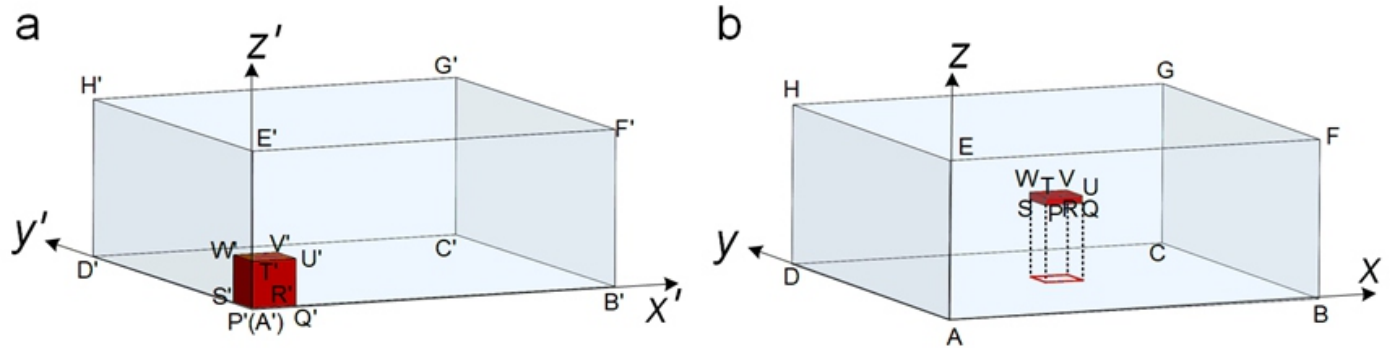

C

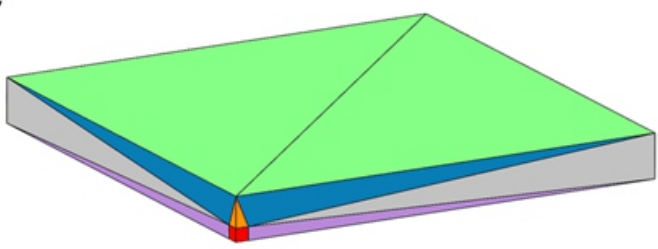

d

e
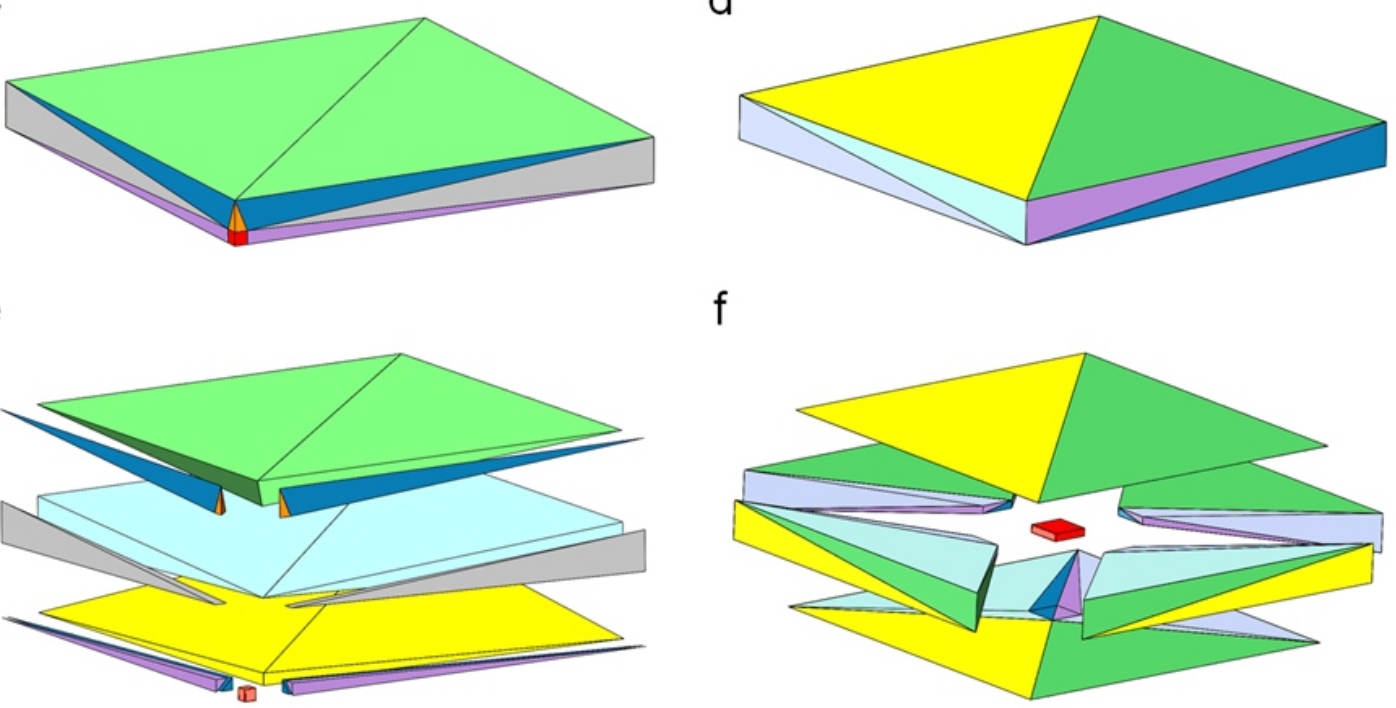

f

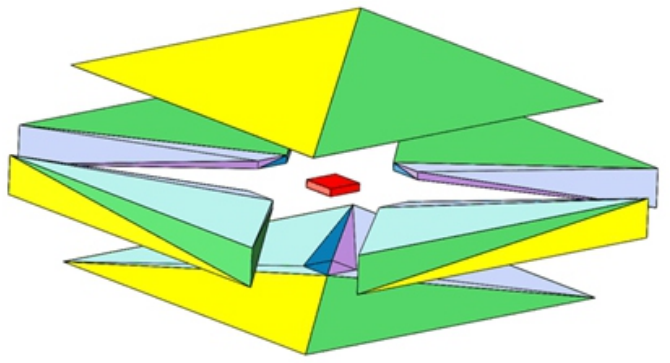

Fig. 1 Schematic of the 3D illusion thermotics design process. (a) Schematic of the first-quadrant plate in the real space with the real heat source located at the original point. (b) Schematic of the first-quadrant plate in the virtual space with the illusion heat source located at a preset position. Schematic of the tetrahedron regions of the real first-quadrant plate in (c) assembly and (e) exploded views. Schematic of the tetrahedron regions of the virtual firstquadrant plate in (d) assembly and (f) exploded views. 


\section{Results and Discussions}

In our design, the dimension of the quarter plate in the first quadrant is length $L=200 \mathrm{~mm}$, width of $W=200 \mathrm{~mm}$ and height of $H=20 \mathrm{~mm}$. The real heat source is located at the origin with dimension of $l^{\prime}=6$ $\mathrm{mm}, w^{\prime}=6 \mathrm{~mm}$, and $h^{\prime}=6 \mathrm{~mm}$. The dimension of the illusion heat source is $l=20 \mathrm{~mm}, w=20 \mathrm{~mm}$ and $h=1 \mathrm{~mm}$ without rotation. In our model, the cuboid box can be divided into 18 tetrahedron regions. For better illustration, Figs. $1 \mathrm{c}$ and $1 \mathrm{e}$ show the assembling and the explosion of the regions of the real plate respectively, Figs. 1d and if show the assembling and the explosion of the regions of the virtual plate respectively. The coordinates of the thermal illusions can be positioned by point $\mathrm{P}$ at $\left(90,90, \mathrm{z}_{\mathrm{p}}\right) \mathrm{mm}$, where $\mathrm{z}_{\mathrm{p}}$ is the $\mathrm{z}$-coordinate of point $\mathrm{P}$ and will be adjusted as follows. Taking the green tetrahedron region as an example, the corresponding Jacobian matrix and the thermal

conductivity tensor are $\left[\begin{array}{lll}1 & 0 & 5.4737 \\ 0 & 1 & 5.4737 \\ 0 & 0 & 0.7368\end{array}\right]$ and $\left[\begin{array}{cccc}42.0188 & 40.6617 & 5.4737 \\ 40.6617 & 42.0188 & 5.4737 \\ 5.4737 & 5.4737 & 0.7368\end{array}\right]$

$\mathrm{W} / \mathrm{m}-\mathrm{K}$ respectively when the $z$-coordinate of point $\mathrm{P}$ is zero. The detailed coordinate transformation can be referred in the Supporting Materials. Following this procedure, we maintain the illusion size and move the illusion heat source to test the $z$-coordinate dependence of the three principal components of thermal conductivity tensor $\left(\kappa_{x x}^{\prime}, \kappa_{y y}^{\prime}\right.$, and $\left.\kappa_{z z}^{\prime}\right)$. It is seen in Figs. $2 \mathrm{a}$ and $2 \mathrm{~b}$ that with the increase of $z_{\mathrm{p}}$, the thermal conductivities of the first layer in Fig. 1e increase gradually. Also, the $\kappa_{x x}^{\prime}$ and $\kappa_{v y}^{\prime}$ are much larger than $\kappa_{z z}^{\prime}$, thus heat tends to diffuse along the $\mathrm{x}-\mathrm{y}$ plane. While the thermal conductivities of the second and third layers in Fig. 1e are almost independent on the z-coordinate of point $\mathrm{P}$, as shown in Figs. 2c-2f. When we focus on $\kappa_{z z}^{\prime}$, it is found that $\kappa_{z z}^{\prime}$ is much larger in the bottom layer, corresponding to stronger heat conduction along the z-direction, and become smaller in the top layer, corresponding to the stronger heat conduction along the $x-y$ plane. The joint outcome of the varying thermal conductivity anisotropy along the heat conduction is the off-lying thermal illusions.

To further test the influence of thermal conductivity anisotropy and height of thermal illusion (z-coordinate of point $\mathrm{P}, z_{\mathrm{p}}$ ) on the thermal camouflage performance, we do the FEM simulations with corresponding calculated thermal conductivity tensors. In the FEM simulation, the heat source is located at the original point with 10 -watt power. The anisotropic thermal conductivities of the 18 tetrahedron regions are calculated according to Eq. (3) independently. All the boundaries are cooled by natural convection with convection coefficient of $5 \mathrm{~W} /\left(\mathrm{m}^{2} \cdot \mathrm{K}\right)$ and ambient air temperature at $20{ }^{\circ} \mathrm{C}$. The simulation a
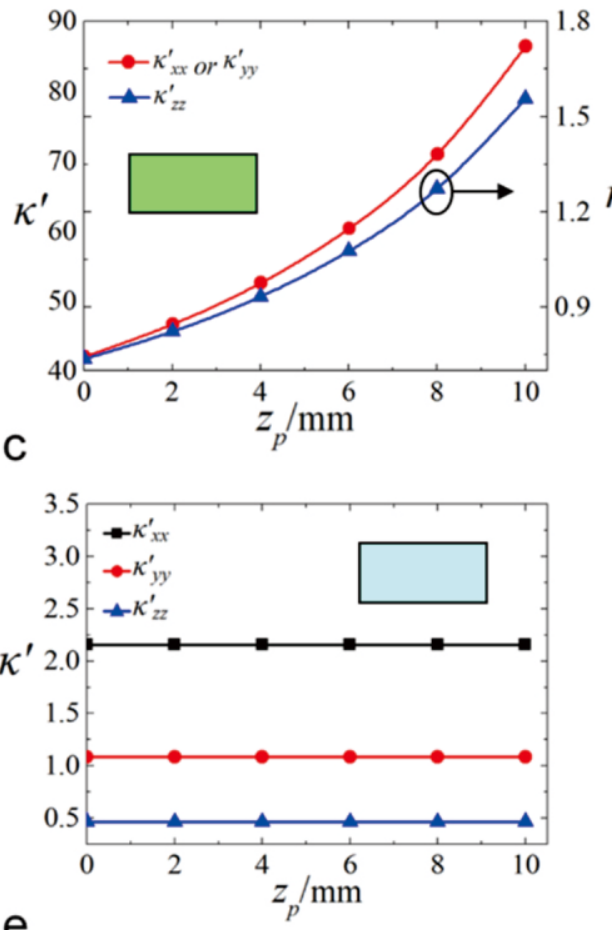

e

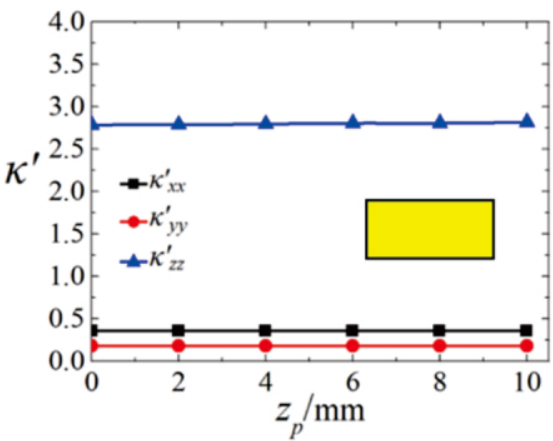

b
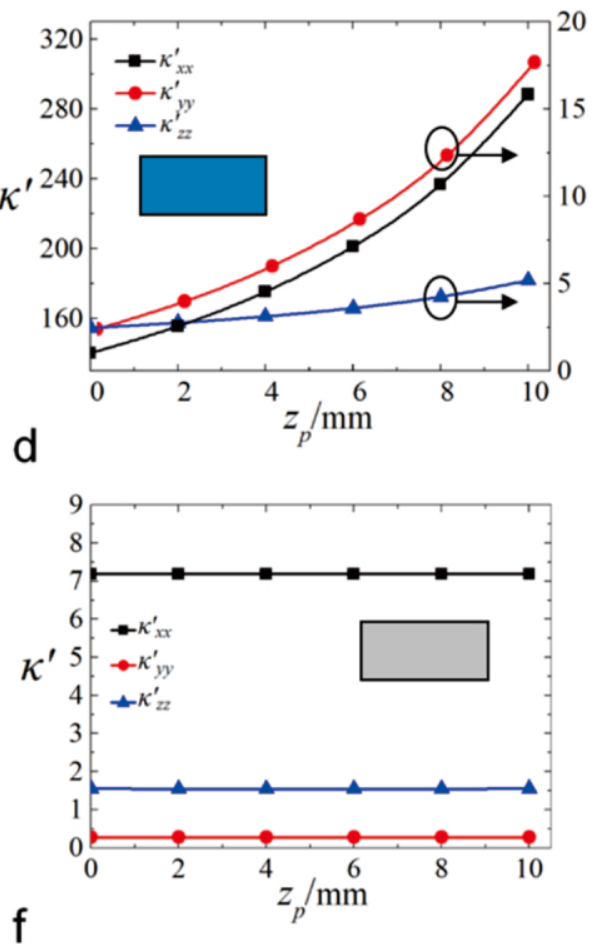

$\mathrm{f}$

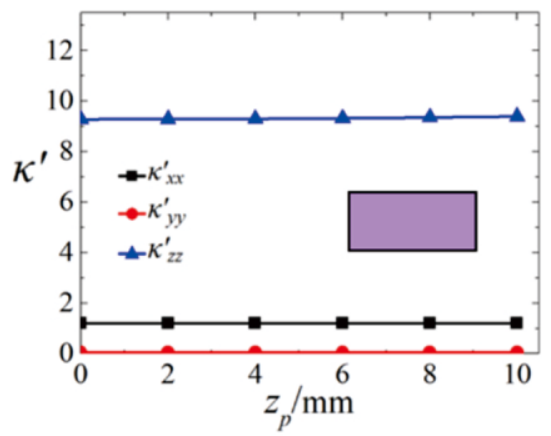

Fig. 2 Dependence of the three principal components of thermal conductivity tensors $\left(\kappa_{x x}^{\prime}, \kappa_{y y}^{\prime}\right.$, and $\left.\kappa_{z z}^{\prime}\right)$ of different tetrahedron regions on the z-coordinate of point P. The color in each subfigure denotes the corresponding tetrahedron region in Fig. 1e. 
results are shown in Fig. 3. From the 3D demonstration, although the real heat source is located at the original point, an off-lying thermal illusion is observed on the top surface (observed plane) of the simulation plate. Therefore, from the IR vision on the top plane, the heat source is located at somewhere off the original point, and the expected thermal camouflage performance is achieved. To examine the z-coordinate dependence, we cut the plate diagonally and redraw the cross sections in Fig. 3c. It is seen that with the increase of $z_{\mathrm{p}}$ from 0,2 , $4,6,8$, to $10 \mathrm{~mm}$, more heat is conducted from the heat source to the thermal illusion. Let's define a temperature difference as the difference of the maximum temperature (point $\mathrm{M}$ ) and the central temperature (point N), i.e. $\Delta T=T_{\mathrm{M}}-T_{\mathrm{N}}$. It is seen in Fig. $3 \mathrm{~d}$ that the increase of $z_{\mathrm{p}}$ leads to larger temperature difference $\Delta T$, implying more obvious thermal illusion and better thermal camouflage performance. It is also noticed that since the position of point $\mathrm{P}$ remains unchanged in the $x-y$ plane, the point $\mathrm{M}$ remains invariant as well. Further validations unfold that the position of point $\mathrm{M}$ depends on the preset position of point $\mathrm{P}$ in the $x-y$ plane.

Note that previous discussions are only based on the quarter plate, we can design the whole plate in different quadrants independently to achieve flexible thermal illusions with different number, size, and position information. As a comparison, the simulated temperature field of a homogenous plate is shown in Fig. 4a, in which the real heat source is located at the center on the bottom plane. Due to the isotropic heat conduction, both the hot spot (maximum temperature point) on the top and bottom planes are located at the center without changing the location and number of hot spots. We first design the whole plate with four symmetric thermal illusions, as shown in Fig. 4b. It is seen that although the real heat source is located in the center bottom of the plate, four off-lying thermal illusions are observed from the top surface of the plate instead, misleading the observers for the wrong number, size, position information of the real heat source. Due to the symmetric design, the temperature field on the bottom plate is also symmetric. The B-B cut-plane shows that heat is conducted to the thermal illusions from the central real heat source. Further, four asymmetric thermal illusions are designed at $(100,100),(-40,130),(-70,-55)$ and $(130,-$ 130) $\mathrm{mm}$, respectively. The FEM simulation results in Fig. $4 \mathrm{c}$ show that the four off-lying asymmetric thermal illusions. Due to the asymmetric design, the temperature field on the bottom plate is also asymmetric. The C-C cut-plane shows that heat is conducted to the thermal illusions from the central real heat source even though the thermal illusions are not symmetric. We also can achieve three illusion heat sources, as shown in Fig. 4d, in which the three thermal illusions are located at $(100,0),(-130,130)$, and $(-70,-150) \mathrm{mm}$, respectively. It is validated that no matter the illusion heat sources are distributed symmetrically or asymmetrically, most heat is conducted to the thermal illusions to achieve perfect thermal camouflage performance with wrong number, size, and position information of the heat source. From the bottom temperature fields, we can see the distorted temperature fields, which are similar to the results of the 2D illusion thermotics and the thermal illusions are not separated. ${ }^{4,18}$ In contrast, we can see the separated thermal illusions from the top temperature fields, which are the right advance of the 3D illusion thermotics with enhanced camouflage performance. a

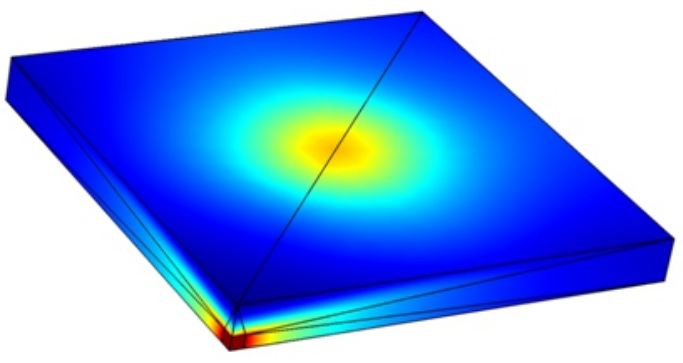

C

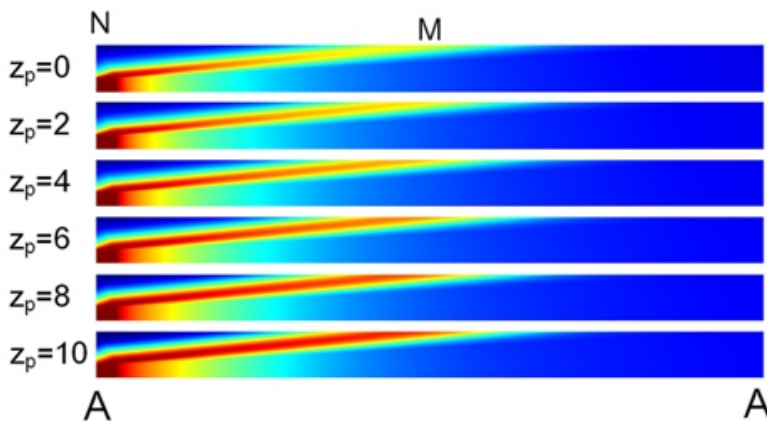

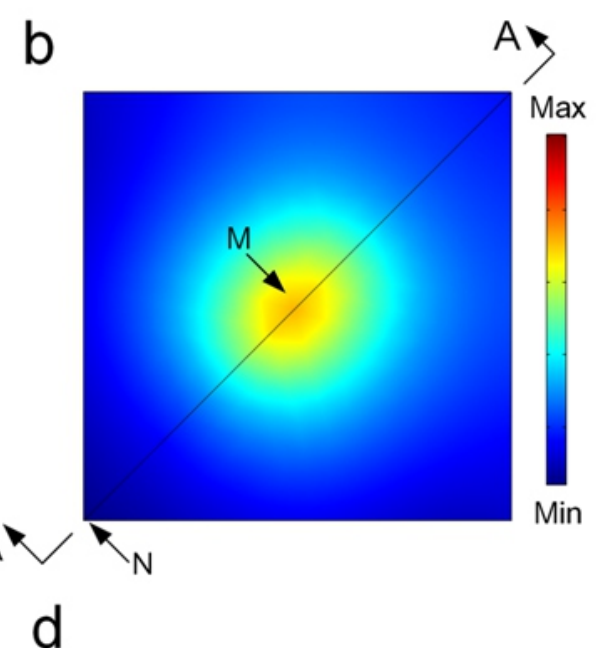

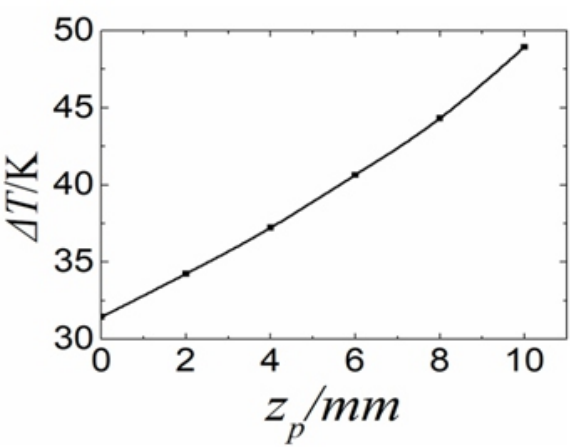

Fig. 3 FEM demonstration of thermal camouflage in the first-quadrant plate. (a) 3D temperature field of the first-quadrant plate and (b) corresponding temperature field of top surface. Point $\mathrm{M}$ denotes the maximum temperature and point $\mathrm{N}$ denotes the corner temperature. (c) Variation of the A-A cutplane temperature field with the increase of z-coordinate of point P. (D) Dependence of the temperature difference of point $\mathrm{M}$ and point $\mathrm{N}$ on the $\mathrm{z}$ coordinate of point $\mathrm{P}$. 
a
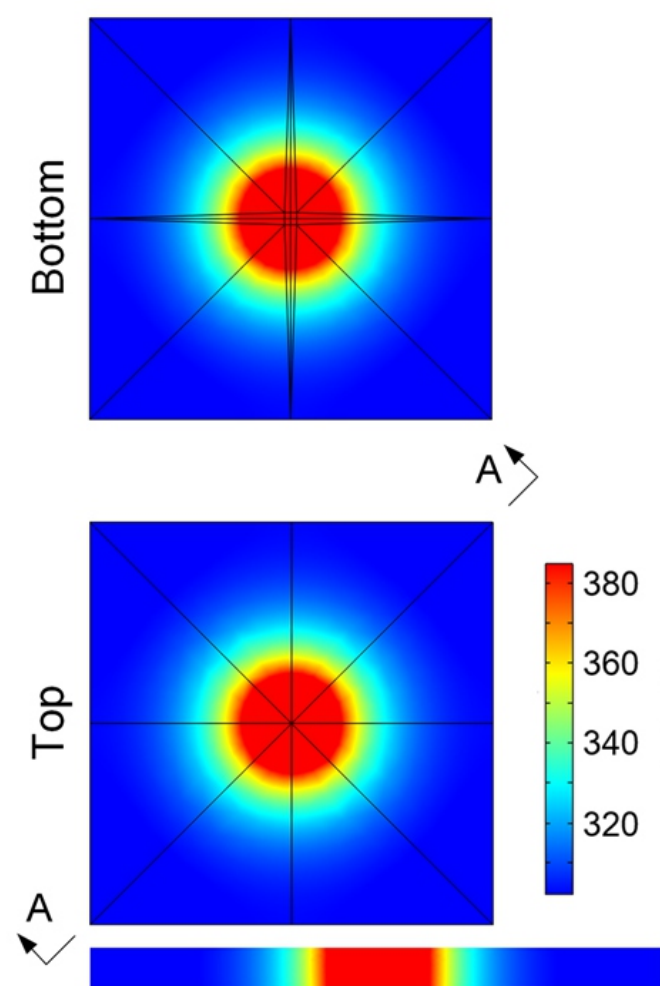

C

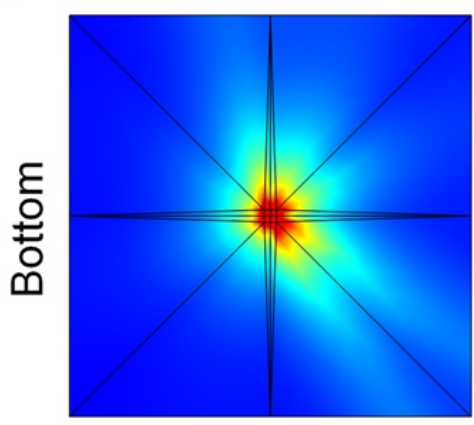

$\mathrm{C}$

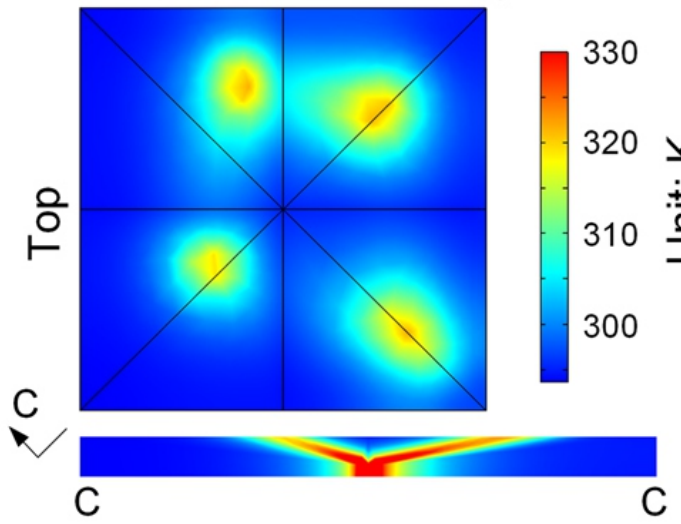

b

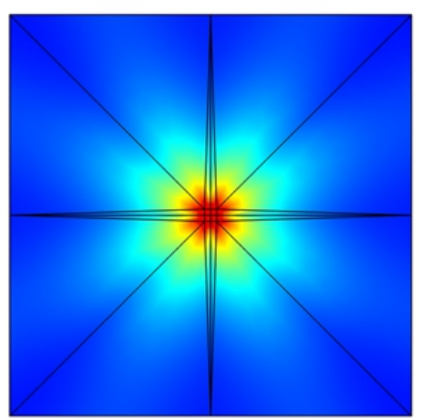

B)

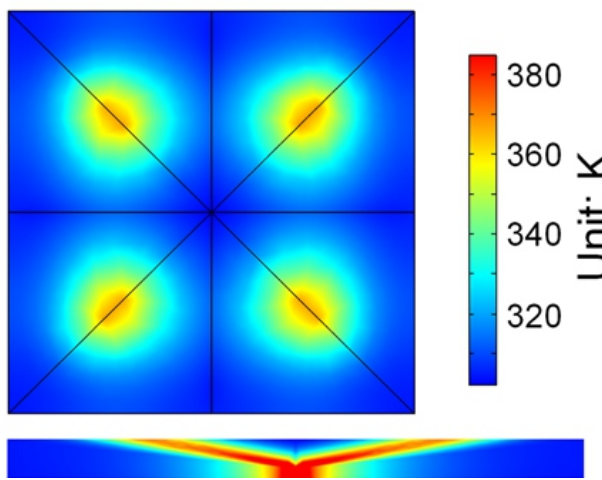

d
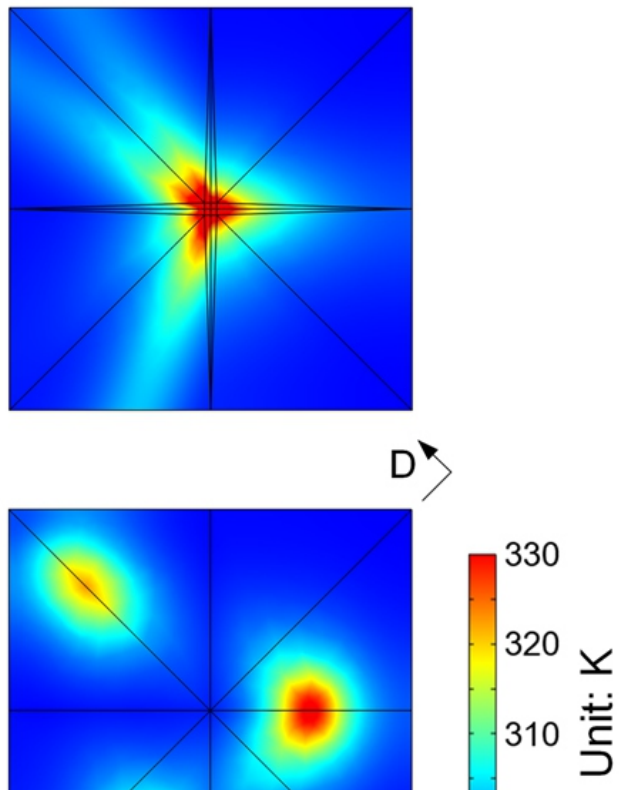

300

Fig. 4 FEM demonstration of the thermal camouflage in whole plate. (a) Top and bottom temperature field of a homogenous plate with a centered heat source. (b-d) Top and bottom temperature field of an anisotropic plate with a centered heat source and multiple illusion heat sources. Through the 3D illusion thermotics design, perfect thermal camouflage performances are achieved by misleading the number, size, and position information of the offlying separated illusion heat sources. 
Compared with the 2D illusion thermotics, the 3D version advances the thermal camouflage performance in following two aspects. 1) Separated thermal illusions. Although we add the splitting function in the $2 \mathrm{D}$ illusion thermotics and expect to observe separated thermal illusions inside the illusion device, heat is conducted from the real heat source to the thermal illusions, whose temperature can approximate but not exactly equal to the real heat source temperature due to the heat loss along the conduction way. In the IR image, a distorted signature of the real source can be observed without separated thermal illusions, thus the real heat source is not camouflaged regardless the perfect camouflage of the external temperature field. Therefore, the separated thermal illusions in the 3D illusion thermotics remove this drawback and enable the achieving of thermal camouflage. 2) Enhanced flexibility. Note that 3D version adds one degree of freedom than the $2 \mathrm{D}$ version, thus the design flexibility can be enhanced. For instance, we can move the designed thermal illusions at different $\mathrm{z}$-coordinates to tune the heat flow inside the 3D architecture. Along with the advance, the manufacturing complexity becomes a bottleneck of experimental demonstration. As shown in this study, the plate is divided into 18 tetrahedron regions and each tetrahedron region has its own anisotropic thermal conductivity tensor, which increases the difficulties of practical manufacturing greatly. 3D printing of composite materials may be one of the solutions. For instance, the diameter, length, orientation, and distribution of the 3D printed copper column array can be adjusted to tune the thermal conductivity. But how to connect these copper columns into a complete object remains an issue. Further simplifying the manufacturing and exploring experimental demonstration will be our next goal in future work.

\section{Conclusions}

In this study, we extend the illusion thermotics from the $2 \mathrm{D}$ version to the $3 \mathrm{D}$ version, based on which we design a conductive plate with anisotropic thermal conductivity tensors to achieve improved thermal camouflage performance. Detailed design process is introduced, and the conductive plate is divided into 18 tetrahedron regions to calculate the required thermal conductivities. Finite-element simulations validate that although the real heat source is located at the bottom center, multiple separated thermal illusions are observed on the top surface, from which the location of the real source is concealed and camouflaged by the offlying thermal illusions. The separated thermal illusions overcome the "linked" drawback of the 2D illusion thermotics, and can mislead the number, size, and position information of the real heat source beautifully with improved thermal camouflage performance. The 3D illusion thermotics can be used to achieve more thermal functionalities with enhanced flexibility at the cost of enhanced complexity in design and manufacturing.

\section{Acknowledgements}

The authors would like to acknowledge the financial support by National Natural Science Foundation of China (51606074, 51625601), and the Ministry of Science and Technology of the People's Republic of China (2017YFE0100600).

\section{References}

1. R. Hu and X. B. Luo, Natl. Sci. Rev., 2019, nwz114, DOI: 10.1093/nsr/nwz114.

2. C. Xu, G. T. Stiubianu and A. A. Gorodetsky, Science, 2018, 359, 1495-1500

3. S. A. Morin, R. F. Shepherd, S. W. Kwok, A. A. Stokes, A. Nemiroski and G. M. Whitesides, Science, 2012, 337, 828-832.

4. S. A. Cummer, J. Christensen and A. Alu, Nat. Rev. Mater., 2016, 1(3) 16001.

5. R. Hu, S. Zhou, Y. Li, D. Y. Lei, X. Luo and C. W. Qiu, Adv. Mater., 2018, 30, 1707237

6. C. Yu, Y. Li, X. Zhang, X. Huang, V. Malyarchuk, S. Wang, Y. Shi, L. Gao, Y. Su, Y. Zhang, H. Xu, R. T. Hanlon, Y. Huang and J. A. Rogers, Proc. Natl. Ameri. Soc., 2014, 111(36), 12998-13003.

7. K. Iwaszczuk, A. C. Strikewerda, K. Fan, X. Zhang, R. D. Averitt and P. U. Jepsen, Opt. Express, 2012, 20(1), 635-643.

8. T. Han, X. Bai, J. T. L. Thong, B. Li and C. W. Qiu, Adv. Mater, 2014, 26, 1731-1734.

9. Y. Li, X. Bai, T. Yang, H. Luo and C. W. Qiu, Nat. Commun., 2018, 9, 273.

10. X. Huang, G. Liu and X. Wang, Adv. Mater, 2012, 24(11), 1482-1486.

11. B. M. Suleiman, J. Larfeldt, B. Leckner and M. Gustavsson, Wood Sci. Technol., 1999, 33(6), 465-473.

12. N. Song, D. Jiao, S. Cui, X. Hou, P. Ding and Y. Shi, ACS Appl. Mater. Inter, 2017, 9(3), 2924-2932.

13. Y. Chao, B. Duan, L. Li, B. Xie, M. Huang and X. Luo, ACS Appl. Mater. Inter, 2015, 7(23), 13000-13006.

14. C. Z. Fan, Y. Gao and J. P. Huang, Appl. Phys. Lett., 2008, 92, 251907.

15. S. Guenneau, C. Amra and D. Veynante, Opt. Express, 2012, 20(7), 82078218.

16. S. Zhou, R. Hu and X. Luo, Int. J. Heat Mass Transf., 2018, 127, 607-613.

17. T. C. Han, C. W. Qiu and X. H. Tang, Opt. Lett., 2011, 36(2), 181-183.

18. T. Han and Z. Wu, Opt. Lett., 2014, 39(13), 3698-3701.

19. L. Zhou, S. Huang, M. Wang, R. Hu and X. Luo, Phys. Lett. A, 2019, 383, 759-763.

20. R. Hu, S. Huang, M. Wang, L. Zhou, X. Peng and X. Luo, Phys. Rev. Appl., 2018, 10, 054032

21. Y. Liu, Y. Cheng, R. Hu and X. Luo, Phys. Lett. A, 2019, 383(19), 22962301.

22. R. Hu, S. Huang, M. Wang, X. Luo, J. Shiomi and C. W. Qiu, Adv. Mater, 2019, 31, 1807849

23. T. Han, X. Bai, D. Gao, J. T. L. Thong, B. Li and C. W. Qiu, Phys. Rev. Lett., 2014, 112, 054302

24. L. J. Xu and J. P. Huang, Phys. Rev. E 2019, 99, 042144

25. L. J. Xu, R. Z. Wang and J. P. Huang, J. Appl. Phys. 2018, 123, 245111.

26. R. Z. Wang, J. Shang and J. P. Huang, Int. J. Therm. Sci. 2018, 131, 14-19.

27. T. C. Han, P. Yang, Y. Li, D. Y. Lei, B. W. Li, K. Hippalgaonkar and C. W. Qiu, Adv. Mater. 2018, 30, 1804019.

Publisher's Note Engineered Science Publisher remains neutral with regard to jurisdictional claims in published maps and institutional affiliations. 